\title{
77-GHz SAR Measurements with Integrated Sensors
}

Schrattenecker J., Haderer A., and Stelzer A.

Institute for Communications Engineering and RF-Systems

Johannes Kepler University, Altenberger Str. 69, A-4040 Linz, Austria

\section{Introduction}

Recent advances enable the realization of low-cost and high performance mm-wave radar sensors suitable for miscellaneous applications. In this contribution, a $77-\mathrm{GHz}$ frequency-modulated continuous-wave (FMCW) radar is used to realize a mm-wave imaging system. When dealing with distances up to a few hundred meters, FMCW radar systems gained increased importance due to their simple and cheap architecture. In addition, these sensors are robust against environmental stress like fume, dust, etc. Hence, they are of great importance in many industrial applications, e.g. filling level measurements or speed and range measurements in automotive usage.

Radar systems with a single turnable antenna have limited angular resolution capabilities, which depend on the directivity of the antenna. Therefore, objects located at the same range but at distinct angles of observation can not be identified as separate targets in general. This major disadvantage can be overcome by conducting measurements at spatially separated locations, which is known as synthetic aperture radar (SAR). At the expense of an increased measurement time, high resolution images of the illuminated scene can be reconstructed. In our work, the actual image formation is done offline by means of digital signal processing, where the measured data are coherently accumulated. When operating SAR systems at mm-wave frequencies, cross-range resolutions of a few millimeters can be achieved.

\section{FMCW PRINCIPLE}

The basic functionality of an FMCW radar [1] can be explained with a simplified schematic of a quasi monostatic architecture, as shown in the block diagram in Fig. 1. The transmit part consists of a voltage controlled oscillator (VCO), which generates the transmit signal. Commonly, FMCW radars utilize a linear frequency modulation, as shown in the right plot of Fig. 1. For an up-chirp the transmit frequency $f_{t}(t)$ linearly increases from the start frequency $f_{0}$ to the stop frequency $f_{0}+B$, where $B$ denotes the bandwidth of the transmit signal. The complex transmit signal for a single up-chirp can be written as

$$
s_{\mathrm{t}}(t)=A_{\mathrm{t}} \mathrm{e}^{\mathrm{j} 2 \pi\left(f_{0} t+\frac{k_{\mathrm{f}}}{2} t^{2}\right)},
$$

where $A_{\mathrm{t}}$ denotes the transmit signal's amplitude. The chirp rate is denoted by $k_{\mathrm{f}}=B / T$ and specifies the slope of the linearly increasing frequency and $T$ refers to the ramp duration. In order to state a simple signal model, we assume that a complex target can be modeled with $P$ independent, dominant point scatterers. The transmit signal $s_{\mathrm{t}}(t)$ is radiated by the antenna and after the round trip delay time (RTDT)

$$
\tau_{i}=\frac{2 R_{i}}{c_{0}}
$$

the backscattered part, due to the $i$ th target arrives at the radar's receive antenna. In (2), $R_{i}$ describes the distance between the antenna and the $i$ th target and $c_{0}$ is the propagation velocity of the electromagnetic wave in free space. Thus, the receive signal for the $i$ th target

$$
s_{\mathrm{r}, i}(t)=\rho_{i} s_{\mathrm{t}}\left(t-\tau_{i}\right),
$$

is a damped and delayed version of the transmit signal. The attenuation of the electromagnetic wave, propagating in free space, and the radar cross section (RCS) of the $i$ th target are included in the factor $\rho_{i}$. Due to the RTDT, the instantaneous frequency $f_{\mathrm{r}, i}(t)$ of the received signal is time shifted with respect to the transmit frequency $f_{\mathbf{t}}(t)$. 

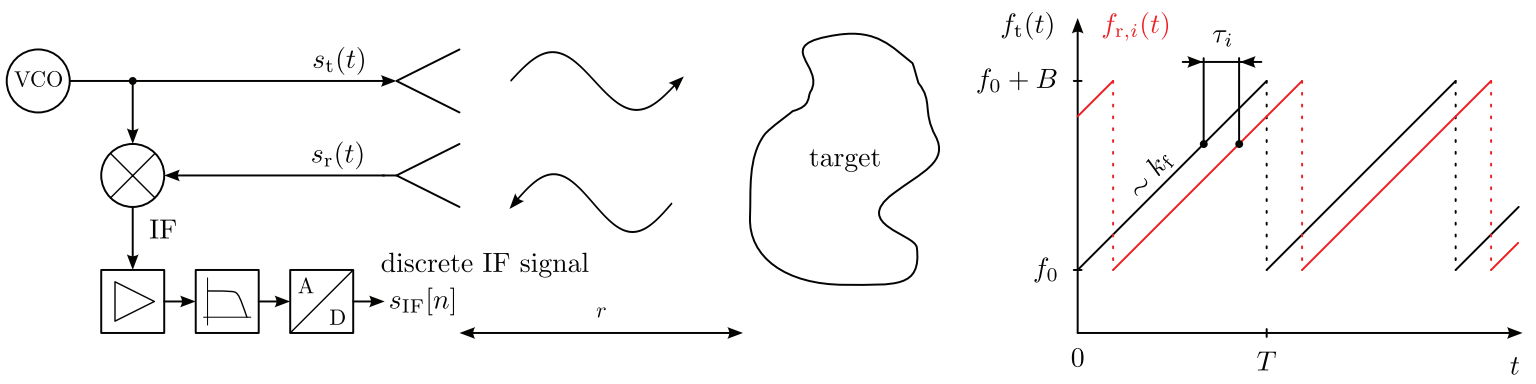

Figure 1: The left figure shows a simplified schematic of an FMCW radar and the right plot depicts the instantaneous transmit and receive frequencies for consecutive up-chirps.

The received signal is down converted with a part of the transmit signal. Thus, the time shift is transfered into a frequency shift, which is denoted as de-ramping. The mixer's output, the intermediate frequency (IF) signal,

$$
s_{\mathrm{IF}}(t)=\sum_{i=1}^{P} K \rho_{i} A_{\mathrm{t}}^{2} e^{-\mathrm{j} 2 \pi f_{0} \tau_{i}-\mathrm{j} 2 \pi k_{\mathrm{f}} \tau_{i} t},
$$

is the sum of the contributions from the $P$ point targets and is used for further signal processing. Additionally, the residual video phase (RVP) term is neglected, because of its low influence for short distances. In (4), $K$ refers to the mixer's gain. The distance information is contained in both, the frequency and the phase of the IF signal. Using the frequency information, the distance to the $i$ th target can be calculated via the relation

$$
R_{i}=\frac{f_{\mathrm{IF}, i} c_{0}}{2 k_{\mathrm{f}}}
$$

where $f_{\mathrm{IF}, i}=k_{\mathrm{f}} \tau_{i}$. Hence, the distance can be calculated by means of frequency estimation. For the SAR principle, as it is used in this work, the phase term plays an important role, too. The range dependent phase of the IF signal is used to focus the reconstructed image in cross-range direction.

\section{SAR PRINCIPLE}

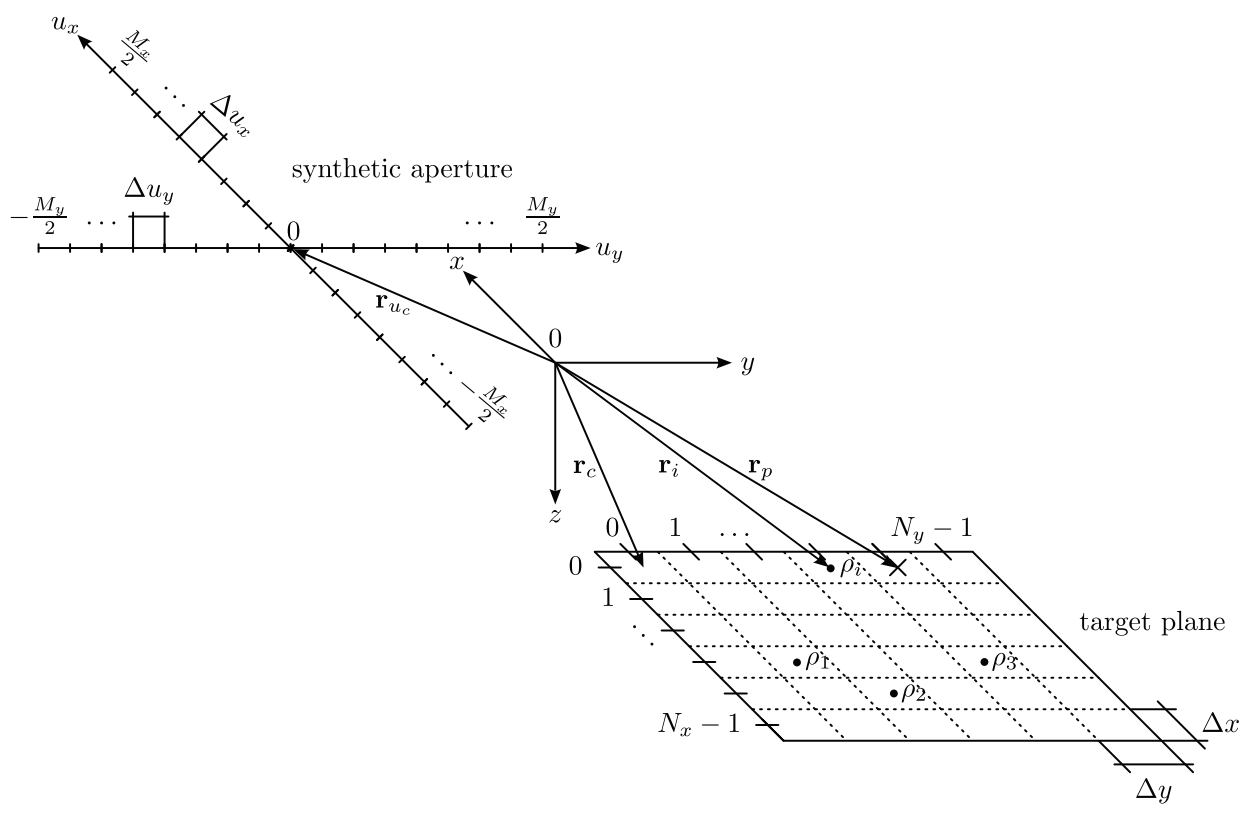

Figure 2: Geometry of a two-dimensional synthetic aperture. 
A synthetic aperture can be used to reconstruct high resolution images of the illuminated target scene [2]. Commonly, such systems are used for monitoring, surveying, and reconnaissance tasks. The SAR principle requires a relative movement between the radar and the targets. Fig. 2 shows the geometry of a two-dimensional SAR system. As sketched in the figure, the synthetic aperture spans from $-L_{x}$ to $L_{x}$ in the $x$ - and from $-L_{y}$ to $L_{y}$ in the $y$-direction. In order to derive a simple but adequate signal model, we only assume stationary targets. In this case an FMCW SAR IF signal can be derived from geometrical considerations. The distance between the $i$ th target and the radar antenna is given as

$$
R_{i}=\left|\mathbf{r}_{i}-\mathbf{r}_{u}\right|,
$$

where $\mathbf{r}_{i}$ represents the vector to the $i$ th target and $\mathbf{r}_{u}$ specifies the position of the antenna along the synthetic aperture. When using (6) in (4) a signal model

$$
s_{\mathrm{IF}, \mathrm{C}}\left(k_{r}, \mathbf{r}_{u}\right)=\sum_{i=1}^{P} A_{\mathrm{IF}, i} D\left(\mathbf{r}_{u}, \mathbf{r}_{i}\right) e^{-\mathrm{j} 2 k_{r}\left|\mathbf{r}_{i}-\mathbf{r}_{u}\right|},
$$

for an FMCW SAR is found. In (7), $A_{\mathrm{IF}, i}$ denotes the amplitude of the $i$ th target and $D\left(\mathbf{r}_{u}, \mathbf{r}_{i}\right)$ accounts for the radiation characteristic of the antenna. The radiation characteristic plays an important role in SAR systems, by contrast to non moving radar systems as presented in section I. Furthermore, $k_{0}=\frac{2 \pi f_{0}}{c_{0}}$ denotes the wave number at the start frequency and $k_{r}=k_{0}+\frac{2 \pi k_{f} t}{c_{0}}$ refers to the wave number as a function of time.

In a real measurement setup, the continuous signal is sampled. The IF signal is sampled in the time domain with sampling frequency $f_{s}=\frac{1}{T_{s}}$. In the spacial domain the signal is sampled because of the discrete antenna positions. The discretized IF signal can be calculated with the relations

$$
\begin{array}{ll}
k_{r}=k_{0}+\Delta k_{r} m_{r} & m_{r}=0,1, \ldots M_{r}-1 \\
u_{x}=\Delta u_{x} m_{x} & m_{x}=-\frac{M_{x}}{2},-\frac{M_{x}}{2}+1, \ldots, \frac{M_{x}}{2} \\
u_{y}=\Delta u_{y} m_{y} & m_{y}=-\frac{M_{y}}{2},-\frac{M_{y}}{2}+1, \ldots, \frac{M_{y}}{2},
\end{array}
$$

where $\Delta u_{x}$ and $\Delta u_{y}$ denote the spacial sampling interval in the $x$ - and $y$-direction. The increment $\Delta k_{r}=$ $\frac{2 \pi k_{r} T_{s}}{c_{0}}$ specifies the increase in the wave number, $M_{r}$ the number of samples and $M_{x}+1$ and $M_{y}+1$ the number of positions in the $x$ - and $y$-direction. Due to the discrete positions in the plane of the synthetic aperture the position vector can be stated as

$$
\mathbf{r}_{u}\left[m_{x}, m_{y}\right]=\mathbf{r}_{u_{c}}+\left[\begin{array}{lll}
\Delta u_{x} m_{x} & \Delta u_{y} m_{y} & 0
\end{array}\right]^{\top},
$$

and is available at discrete values. Substituting (8) into (7) leads to the discrete signal model $s_{\mathrm{IF}, \mathrm{d}}\left[m_{r}, m_{x}, m_{y}\right]$.

The size of the synthetic aperture has a limiting impact on the angular resolution of the system. The maximum useful aperture size is given by the antenna's radiation characteristic. Similar to other array imaging systems, a SAR provides a multidimensional database that can be manipulated via signal processing. In our case of a two-dimensional synthetic aperture a three-dimensional IF signal

$$
x\left[m_{r}, m_{x}, m_{y}\right]=s_{\mathrm{IF,d}}\left[m_{r}, m_{x}, m_{y}\right]+\omega\left[m_{r}, m_{x}, m_{y}\right]
$$

is measured. The measured data consists of a sum of the deterministic IF signal $s_{\mathrm{IF,d}}\left[m_{r}, m_{x}, m_{y}\right]$ and the stochastic part $\omega\left[m_{r}, m_{x}, m_{y}\right]$, caused by noise. Commonly, $\omega\left[m_{r}, m_{x}, m_{y}\right]$ is Gaussian distributed. In the next section, an algorithm to reconstruct the area under observation is explained.

\section{IMAGE RECONSTRUCTION}

A correlation algorithm provides an optimal image reconstruction method. In many cases, like edge detection or surface scanning the targets are positioned in the same plane $z=z_{\top}$. Therefore, it is sufficient to 
reconstruct the image of this area. The plane is divided into $N_{x} N_{y}$ pixels with a size of $\Delta x \Delta y$ as shown in Fig. 2. The vector $\mathbf{r}_{c}$ specifies the image's position in a Cartesian coordinate system. The center of each pixel can be described with the vector

$$
\mathbf{r}_{p}\left[n_{x}, n_{y}, z_{\mathrm{T}}\right]=\mathbf{r}_{c}+\left[\begin{array}{lll}
\Delta x n_{x} & \Delta y n_{y} & 0
\end{array}\right]^{\top}
$$

where $n_{x}$ and $n_{y}$ number the different pixels. For each pixel in the image, a reference signal $s_{\mid F, p}\left[m_{r}, m_{x}, m_{y}\right]$ at position $\mathbf{r}_{p}\left[n_{x}, n_{y}, z_{\mathrm{T}}\right]$ and with an amplitude $A_{\mathrm{IF}}=1$, based on $s_{\mathrm{IF}, d}\left[m_{r}, m_{x}, m_{y}\right]$, is calculated. Afterwards, the reference signal is correlated with the measured data $x\left[m_{r}, m_{x}, m_{y}\right]$

$$
\hat{A}_{\mathrm{IF}}\left[n_{x}, n_{x}, z_{\mathrm{T}}\right]=\left.\frac{1}{E} \sum_{m_{x}} \sum_{m_{y}} \sum_{m_{r}} x\left[m_{r}, m_{x}, m_{y}\right] s_{\mathrm{IF}, p}^{*}\left[m_{r}, m_{x}, m_{y}\right]\right|_{\mathbf{r}_{p}} .
$$

This causes an estimation of the amplitude for the different pixels. The variable

$$
E=M_{r} \sum_{m_{x}} \sum_{m_{y}} D_{\mathrm{d}}^{2}\left[m_{x}, m_{y}, n_{x}, n_{y}, z_{\mathrm{\top}}\right]
$$

refers to a scaling factor, which depends on the antenna's characteristic $D_{\mathrm{d}}\left[m_{x}, m_{y}, n_{x}, n_{y}, z_{\mathrm{T}}\right]$, which is a function of the discrete antenna positions and reconstruction positions. Therefore, $E$ is not a constant factor but scales the output of the correlation algorithm depending on the pixels' reconstruction position. To reduce the complexity of the algorithm the inner summation can be calculated by an inverse fast Fourier transform (IFFT)

$$
X\left[R, m_{x}, m_{y}\right]=\sum_{m_{r}} x\left[m_{r}, m_{x}, m_{y}\right] e^{\mathrm{j} 2 \Delta k_{r} m_{r} R} .
$$

for each antenna position $m_{x}$ and $m_{y}$. If (14), is evaluated at

$$
R_{p}=\left|\mathbf{r}_{p}-\mathbf{r}_{u}\right|
$$

and multiplied with the complex balance factor $e^{\mathrm{j} 2 k_{0} R_{p}}$, the result is the same as the inner sum in (12). After calculating the IFFT, the values $X\left[R, m_{x}, m_{y}\right]$ are available on equidistant bins with the spacing $\Delta R$. Because these values do not necessarily fit the needed values $\left.X\left[R, m_{x}, m_{y}\right]\right|_{R=R_{p}}$, an interpolation scheme has to be applied. To achieve a fast and simple interpolation the nearest-neighbor method is used.

\section{RADAR SENSOR AND MEASUREMENT RESULTS}

The SAR measurements were conducted with an FMCW prototype system. A picture and a block diagram of the hardware are shown in Fig. 3. The central processing element, controlling the entire radar system, is built with an Altera Cyclone-III FPGA. The FPGA manages controlling functions only. These can be summarized as follows: enabling and disabling the RF frontend, configuration and control of the ADC, setting the parameters of the synthesizer for the frequency ramp, triggering the start of the frequency ramps and communication with an USB controller.

A synthesizer, consisting of a DDS and a PLL, is used to generate the frequency ramps. Thus, the system is capable of generating user-defined ramp sequences. The measured, and de-ramped signal is amplified by a variable gain amplifier (VGA) with a gain of $15 \mathrm{~dB}$. A 14 Bit ADC with a maximum sample rate of $40 \mathrm{MSamples} / \mathrm{s}$ allows the sampling of the amplified data. After that, the discrete data can be stored in a 32 MB DDR-SDRAM or directly transferred to a host PC via an USB 2.0 compatible interface. 


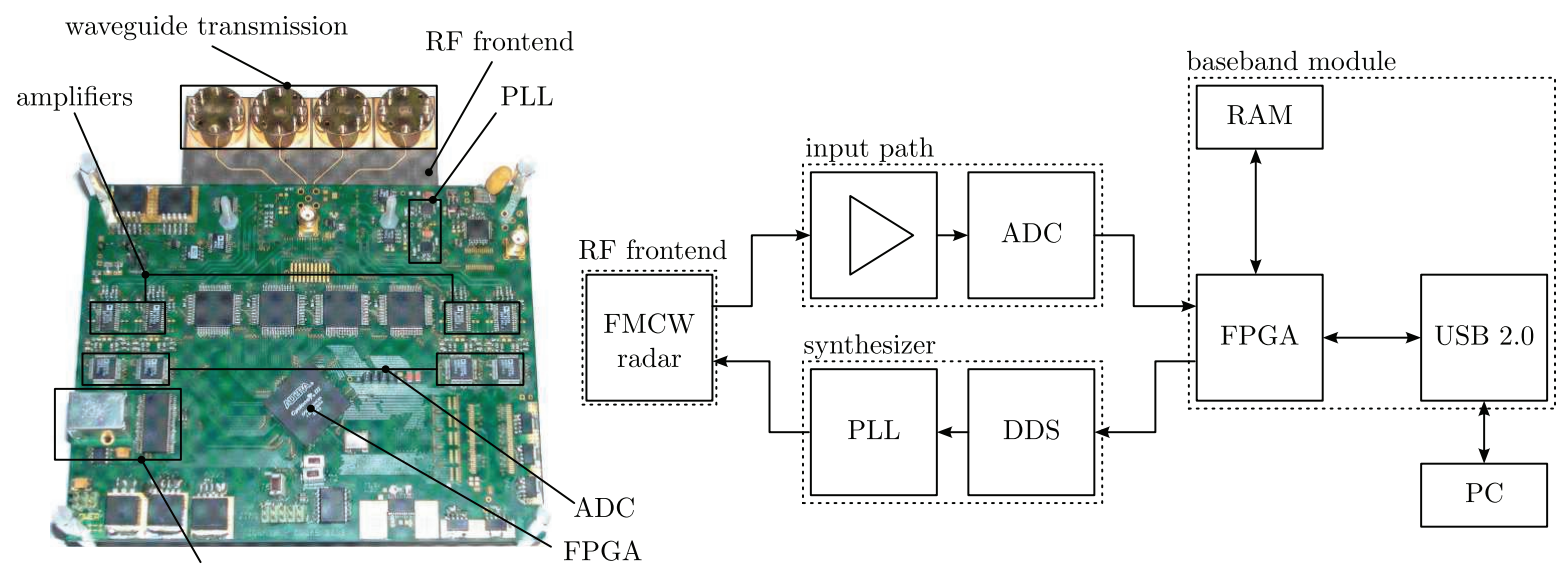

USB 2.0

Figure 3: The picture and the block diagram display the interaction between the different modules. An FPGA controls the synthesizer, required for the generation of the frequency ramps. The receive path consists of an amplifier and an ADC.

The FMCW radar prototype was primarily developed to evaluate different RF frontends [3]. In this particular case a two-dimensional SAR is realized with the prototype system. For this purpose, the sensor was mounted on a cantilever, as shown in Fig. 4. The targets were placed in a distance of $0.72 \mathrm{~m}$ on a sleigh, which can be moved by a portal axes on a two-dimensional grid.
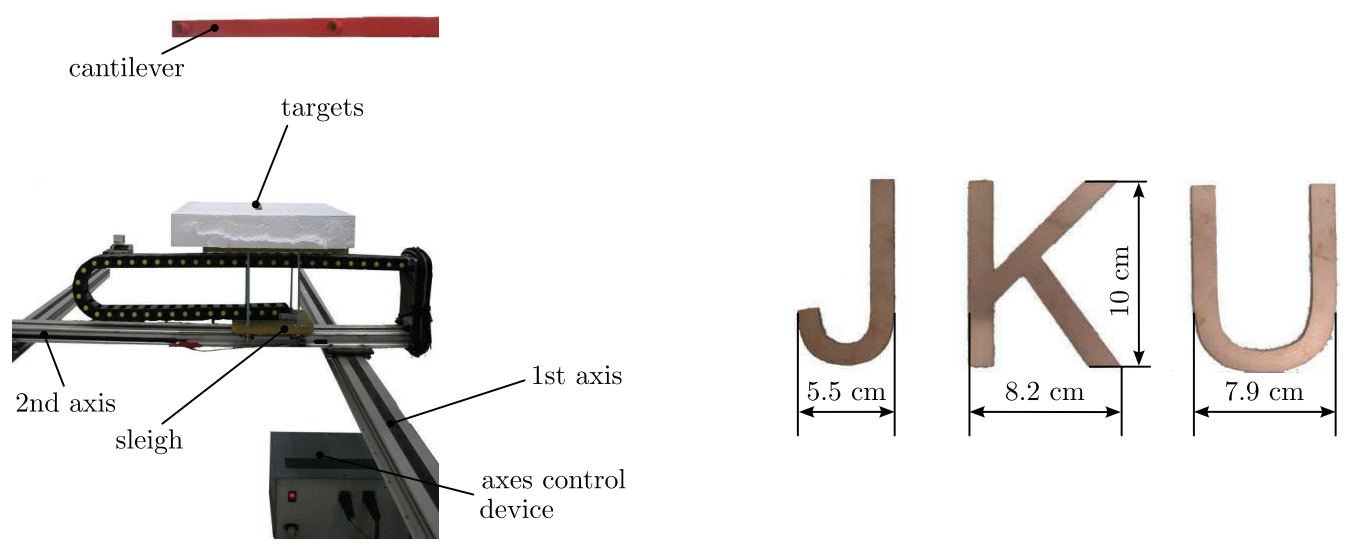

Figure 4: The left photograph shows the portal axes in order to move the targets along the synthetic aperture and the cantilever, where the prototype is mounted. The right photograph shows the scanned JKU letters.

A Matlab ${ }^{\circledR}$ script was used to control the position of the targets, and for conducting measurements. The targets were positioned along the synthetic aperture. Then the measurements were taken. The measured data were transferred to a PC and the signal processing was directly done within a Matlab environment. With this system, a JKU writing made of copper, as shown in Fig. 4, was scanned. A summary of the used SAR parameters is listed in Tab. 1.

With this setup, a cross-range resolution of about $5.0 \mathrm{~mm}$ can be achieved. The left plot in Fig. 5 shows the gathered SAR signal after the IFFT at the aperture-position $u_{x}=u_{y}=0.0 \mathrm{~m}$ normalized to its maximum. The reconstruction of the target area was done with the correlation algorithm as presented section III. The result of the complete reconstruction is shown in the right plot of Fig. 5. The image clearly reveals the measured target's shape with the correct dimensions. 


\begin{tabular}{llll} 
synthetic aperture & \multicolumn{3}{c}{ FMCW radar } \\
\hline \hline aperture length in $x$ & $0.3 \mathrm{~m}$ & antenna gain & $\approx 12 \mathrm{dBi}$ \\
aperture length in $y$ & $0.3 \mathrm{~m}$ & start frequency & $76 \mathrm{GHz}$ \\
sampling space & $2.0149 \mathrm{~mm}$ & bandwidth & $2 \mathrm{GHz}$ \\
radar altitude & $0.72 \mathrm{~m}$ & ramp duration & $100 \mu \mathrm{s}$ \\
& & sampling frequency & $1.5625 \mathrm{MHz}$
\end{tabular}

Table 1: The table lists the parameters of the imaging system.
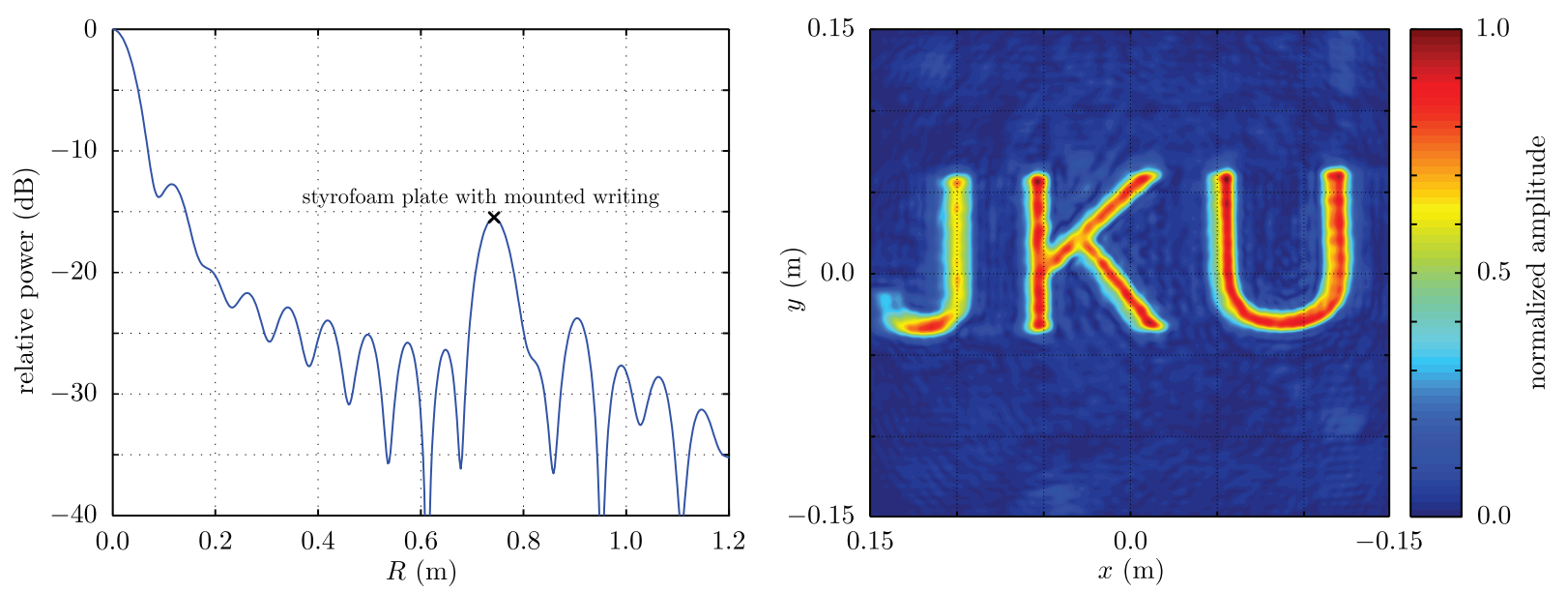

Figure 5: The left plot shows the measured data at the position $u_{x}=u_{y}=0.0 \mathrm{~m}$ after the IFFT. The right plot shows the reconstructed image normalized to the maximum.

\section{CONCLUSION}

In this paper, we described the realization of an FMCW SAR system. The stacked RF frontend provides a high flexibility for use with different frontends. With the presented measurement setup a cross-range resolution of a few millimeters can be achieved. Therefore, and due to the robustness of the FMCW radar, the system can be used for high resolution image measurements of surfaces and automated positioning in environmental stressful areas. The used correlation algorithm allows fast reconstruction of the target area and has very good conditions for parallel calculations.

\section{ACKNOWLEDGMENT}

The authors would like to thank the members of the Christian Doppler Laboratory for Integrated Radar Sensors for providing the FMCW prototype system.

\section{References}

[1] A. G. Stove. "Linear FMCW radar techniques," IEEE Proc. F. Radar and Signal Processing, vol. 139, no. 5, pp. 343-350, Oct. 1992.

[2] M. Soumekh. Synthetic Apertur Radar Signal Processing with MATLAB Algorithms. New York: John Wiley \& Sons INC., 1999.

[3] R. Feger, C. Wagner, S. Schuster, S. Scheiblhofer, H. Jaeger, and A. Stelzer. "A 77-GHz FMCW MIMO Radar Based on an SiGe Single-Chip Transceiver." IEEE Trans. Microw. Theory Tech., vol. 57, pp. 10201035, May 2009. 